\title{
Non-Adherence to Prescribed Antihypertensives in Primary, Secondary and Tertiary Healthcare Settings in Islamabad, Pakistan: A Cross-Sectional Study
}

This article was published in the following Dove Press journal: Patient Preference and Adherence

\author{
Sajid Mahmood (1D' \\ Zahraa Jalal $^{2}$ \\ Muhammad Abdul Hadi (iD ${ }^{2}$ \\ Hasan Orooj ${ }^{3}$ \\ Kifayat Ullah Shah (D) \\ 'Department of Pharmacy, Quaid-e-Azam \\ University, Islamabad 45320, Pakistan; \\ ${ }^{2}$ School of Pharmacy, College of Medical \\ and Dental Sciences, Institute of Clinical \\ Sciences, University of Birmingham, \\ Edgbaston, Birmingham BI5 2TT, UK; \\ ${ }^{3}$ Directorate of Health Services, \\ Metropolitan Corporation Islamabad, \\ Islamabad, Pakistan
}

Objective: This study aimed to assess the prevalence and predictors of non-adherence to antihypertensive medication among patients with hypertension attending various healthcare settings in Islamabad, Pakistan.

Methods: A questionnaire-based cross-sectional study was conducted in selected healthcare facilities between September 2017 and December 2018. The study was conducted in primary, secondary and tertiary healthcare settings in Islamabad, Pakistan. Medication adherence was assessed using the eight-item Morisky Medication Adherence Scale (MMAS-8). Sociodemographic and clinical data of recruited patients were collected through a structured questionnaire. Binary logistic regression analysis was performed to determine covariates significantly associated with medication adherence and blood pressure control.

Results: A total of 776 hypertensive patients were invited and 741 (95\%) completed the questionnaire. The mean \pm SD age of participants was $53.6 \pm 12.6$ years; 284 patients $(38.3 \%)$ had high adherence, 178 (24\%) had moderate adherence and 279 (37.7\%) were non-adherent to the prescribed antihypertensive therapy. Binary regression analysis revealed that old age (OR 1.783 [95\% CI: 1.172-2.712]; $P=0.013$ ), being educated (OR 2.018 [95\% CI: $1.240-3.284]$; $P=0.036$ ), entitlement to free medical care (OR 1.369 [95\% CI: 1.009-1.859]; $P=0.044$ ), treatment duration (OR 2.868 [95\% CI: 1.913-4.299]; $P=0.001$ ), number of medications (OR 1.973 [95\% CI: $1.560-2.495$ ]; $P=0.001$ ), presence of any comorbidity (OR 2.658 [95\% CI: 1.836-3.848]; $P=0.001$ ) and blood pressure control (OR 3.103 [95\% CI: 2.268-4.247]; $P=0.001$ ) were significantly associated with good adherence. Similarly, age (OR 1.998 [95\% CI: 1.313-3.040]; $P=0.004$ ), entitlement to free medical care (OR 1.498 [95\% CI: 1.116-2.010]; $P=0.007$ ), treatment duration (OR 1.886 [95\% CI: $1.143-3.113$ ]; $P=0.013$ ), presence of any comorbidity (OR 1.552 [95\% CI: 1.123-2.147]; $P=0.008$ ) and adherence level (OR 3.103 [95\% CI: 2.268-4.247]; $P=0.001$ ) had significant association with controlled blood pressure. The following were the main reasons for non-adherence to prescribed antihypertensive medication: "don't feel need for regular use" (24.7\%), "Carelessness" (13.4\%) and "adverse effects" (11.2\%).

Conclusion: The prevalence of non-adherence to antihypertensive medications was high in the study population and poor medication adherence could potentially explain poor blood pressure control. Evidence-based targeted interventions on both medication adherence and blood pressure control should be introduced and implemented for better treatment outcomes. Keywords: cross-sectional study, adherence, hypertension, Asia, antihypertensives, blood pressure control, Pakistan

\section{Introduction}

Hypertension is a significant global health challenge and a leading cause of morbidity and mortality. Persistently elevated blood pressure may lead to stroke, myocardial
Correspondence: Kifayat Ullah Shah Department of Pharmacy, Quaid-e-Azam University, Islamabad 45320, Pakistan Tel +92-3325542722

Email kushah@qau.edu.pk 
infarction, congestive heart failure, renal failure and blindness. ${ }^{1,2}$ In 2010, 1.3 billion people in the world were suffering from hypertension, with an annual increase rate of 2.5\%. ${ }^{3}$ According to the World Health Organization (WHO) report published in 2013, the global prevalence of hypertension was $40 \%{ }^{4}$ Annually, 9.4 million people die from either hypertension or its related complications worldwide. ${ }^{4}$ Hypertension control is a significant healthcare challenge for both developing and developed countries. In Australia and the USA, the control rate for hypertension was $24 \%$ and $35 \%$, respectively, in 2014-15..$^{5}$ Hypertension control was even worse in Asia, with India and China having control rates of $7.9 \%{ }^{6}$ and $5.7 \%,{ }^{7}$ respectively. A meta-analysis published in 2018 in Pakistan revealed that the overall pooled prevalence of hypertension in Pakistan was 26.3\% (25.93-26.75\%); subgroup analysis revealed that the urban prevalence of hypertension was higher at $26.6 \%(21.80$ $31.42 \%$ ) as compared to the rural prevalence at $21.1 \%$ (10.18-31.87\%). Similarly, the pooled prevalence was $24.9 \%(19.70-30.28 \%)$ in males and $24.7 \%$ (16.76$32.76 \%$ ) in females. ${ }^{8}$

Poor adherence is a critical barrier to treatment success $^{9-11}$ leading to worsening of disease, increased hospitalization rate and longer hospital stays. The estimated overall global prevalence of non-adherence to antihypertensive therapy is around $30 \%{ }^{12}$ In the USA, $5 \%$ of avoidable hospital admissions are due to poor medication adherence, ${ }^{13}$ costing approximately $\$ 100$ billion annually ${ }^{14}$ in the form of both direct and indirect losses.

Optimal adherence to prescribed antihypertensive therapy may result in substantial reductions in the incidence of myocardial infarction (20-25\%), heart failure ( $>50 \%)$ and stroke (35-40\%). ${ }^{15,16}$ Various barriers to antihypertensive medication adherence have been previously identified and reported in the literature. These include cost of medication, lack of education, forgetfulness, unavailability, too much medication, fear of addiction and possible side effects, and dissatisfaction with the therapy. ${ }^{17}$ Therefore, addressing these barriers is necessary to improve medication adherence.

This study aims to evaluate and compare the degree of medication adherence in hypertensive patients in primary, secondary and tertiary healthcare settings in Islamabad. Previously, three studies were conducted in different tertiary care hospitals in Pakistan to evaluate adherence to medication in hypertensive patients. Of these three studies, two used unvalidated questionnaires to assess medication adherence and had too small sample sizes to draw any meaningful conclusions. ${ }^{18,19}$ The largest study of the three was conducted in 2005 in the city of Karachi in a large tertiary care private hospital, and consisted of 460 hypertensive patients. The Morisky, Green, Levine Medication Adherence Questionnaire ${ }^{20}$ was used to assess adherence. ${ }^{17}$ Hypertension is managed primarily in primary care. In Pakistan, $85 \%$ of patients visit primary healthcare facilities for treatment, whereas only $15 \%$ of patients visit tertiary care hospitals. ${ }^{21,22}$ To date, no study has been conducted in Pakistan to assess the degree of medication adherence among hypertensive patients attending primary and secondary care. To fill this research gap, this study was designed to gather a better understanding of the prevalence of and barriers to medication adherence across all three tiers (primary, secondary and tertiary) of Pakistan's healthcare system. The results of this study could inform policy makers to help develop policies with the aim of improving medication adherence for hypertensive patients in Pakistan.

\section{Materials and Methods Ethics Statement}

The study was approved by Quaid-i-Azam University Bioethics Committee (vide letter no. BFC-FBS-QAU -2018-108 dated 23/10/2018.) In addition, ethical approval was obtained from the bioethical committees/administrations of the respective healthcare settings where the study was conducted (vide letter no. F.1-1/2015/ERB/SZABMU dated 28/08/2017 and no. CDA/DHS-14(1) (63)/2018/1077 dated: 09/10/2018). A licensing agreement to use the copyrighted MMAS-8 was obtained from Donald E. Morisky (294 Lindura Court, Las Vegas, NV 89138-4632, USA; dmorisky@gmail.com). Both informed and written consent was sought from each participant using a consent form before enrollment in the study. Survey confidentiality and anonymity were assured to all enrolled participants.

\section{Study Settings}

This was a multicentre study conducted in one primary, one secondary and one tertiary healthcare setting in Islamabad, the capital of Pakistan. This study was conducted in all three tiers of Pakistan's healthcare system to enable generalization of the data to the Pakistani population.

\section{Study Design and Participants}

This was a cross-sectional study including 741 hypertensive patients. The study took place between September 2017 and December 2018. Eligibility criteria included: all patients who were diagnosed with essential hypertension at any 
time; aged 18 years and above; on at least one antihypertensive medication for the past 6 months; able to communicate in Urdu language; and attending one of the participating healthcare facilities. Hypertensive patients with other comorbidities were also included in our study. Pregnant women, patients with mental disorders such as dementia, and those who could not communicate in Urdu, which is the national language of Pakistan, were not included in this study.

\section{Data Collection}

To assess medication adherence, the Urdu version of the eight-item Morisky Medication Adherence Scale (MMAS8) was used. ${ }^{23-25}$ Since Urdu is the national language and is the most widely spoken and understood language in Pakistan, a validated Urdu translation of MMAS- $8^{26}$ was used to measure medication adherence. The MMAS-8 score can range between 0 and 8 . Participants who scored 8 on MMAS- 8 were considered "adherent", participants with a score between 6 and $<8$ were considered "moderately adherent" and participants with an MMAS- 8 score $<6$ were considered "non-adherent" to their prescribed therapy. ${ }^{25}$ A separate standardized data collection form was used to gather patients' sociodemographic data, medical and medication history, and reason for non-adherence. The questionnaires were piloted on 50 patients to test the acceptability of the questions.

\section{Outcome Measures and Covariates}

The primary and secondary outcome measures were optimal medication adherence and optimal blood pressure control, respectively. The target for controlled blood pressure was defined in accordance with NICE treatment guidelines for the management of hypertension 2011. ${ }^{27}$ For patients who were under 80 years of age, blood pressure $<140 / 90 \mathrm{mmHg}$ was considered controlled, whereas for patients aged 80 years or above, blood pressure $<150$ / $90 \mathrm{mmHg}$ was considered controlled. Similarly, for the patients with both hypertension and diabetes mellitus, blood pressure $<140 / 80 \mathrm{mmHg}$, and for patients with kidney, eye and cerebrovascular damage, blood pressure $<130 / 80 \mathrm{mmHg}$ was considered controlled. The covariates were age, gender, entitlement status, marital status, level of education, profession, smoking, number of antihypertensive medications used, duration of therapy and presence of comorbidities.

\section{Statistical Analysis}

Statistical analyses were performed using SPSS version 21.0. The alpha level of significance for all statistical tests was 0.05 . Binary logistic regression analysis using the default entry method was conducted to identify predictors associated with medication adherence and blood pressure control. For the purposes of binary regression analysis, the patients were divided into two categories, ie either adherent or non-adherent, rather than three categories, ie good adherence, moderate adherence and nonadherence, on the basis of their MMAS- 8 score. Patients who had an MMAS- 8 score $\geq 6$ were categorized as adherent and patients who had an MMAS- 8 score $<6$ were categorized as non-adherent. Correlation and HosmerLemeshow Goodness of Fit tests were performed to select the best prediction model.

\section{Results \\ Patient Characteristics}

A total of 768 hypertensive patients using at least one antihypertensive medication were invited to participate, of whom 741 patients participated in the study (Figure 1). Of these 741 patients, 389 (52.5\%) were male and $352(47.5 \%)$ were female. The mean age of participants was $53.6 \pm 12.6$ years. More than one-third of the participants $(286,38.6 \%)$ were obese. At least one comorbidity was present in 205 patients $(27.7 \%)$ (Table 1). The majority of participants were on a single antihypertensive drug $(303,40.9 \%)$ and the mean number of antihypertensive medications used was 1.7. The mean duration of therapy was 5.7 years and the majority of participants had a duration of therapy $<5$ years $(498,67.2 \%)$. The mean MMAS-8 score was $6.17 \pm 1.8 ; 462$ patients $(62.3 \%)$ scored $>6$ and adherence was categorized as either good or moderate, whereas 279 patients (37.6\%) scored $<6$ and were categorized as poor adherents. The

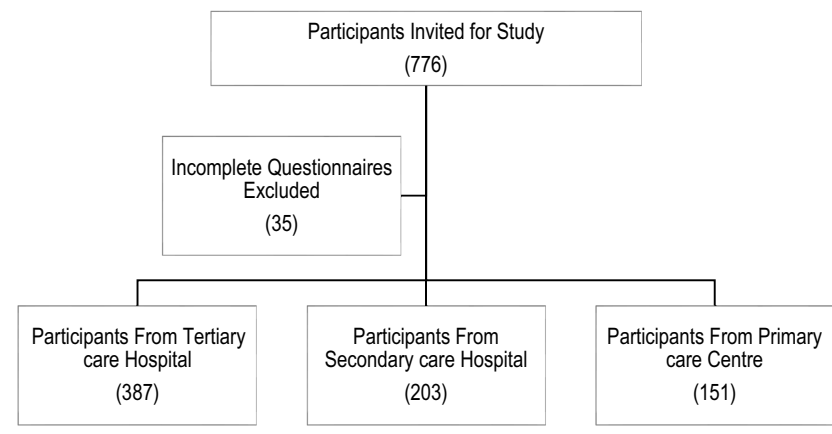

Figure I Flowchart showing the participants from each healthcare setting. 
Table I Characteristics of Patients Included in This Study

\begin{tabular}{|c|c|c|c|}
\hline \multicolumn{2}{|l|}{ Patient Characteristics } & \multicolumn{2}{|c|}{ Total Study Population ( $\mathrm{N}=\mathbf{7 4} \mathrm{I})$} \\
\hline & & $\mathbf{N}$ & $\%$ \\
\hline Gender & $\begin{array}{l}\text { Male } \\
\text { Female }\end{array}$ & $\begin{array}{l}389 \\
352\end{array}$ & $\begin{array}{l}52.5 \\
47.5\end{array}$ \\
\hline Age & $\begin{array}{l}\text { Mean } \pm S D \\
\geq 60 \text { years } \\
40-59 \text { years } \\
\leq 40 \text { years }\end{array}$ & $\begin{array}{l}53.6 \pm 12.6 \\
256 \\
343 \\
142\end{array}$ & $\begin{array}{l}34.5 . \\
46.3 \\
19.2\end{array}$ \\
\hline Marital status & $\begin{array}{l}\text { Married } \\
\text { Unmarried/divorced/widowed }\end{array}$ & $\begin{array}{l}632 \\
109\end{array}$ & $\begin{array}{l}85.3 \\
14.7\end{array}$ \\
\hline BMI & $\begin{array}{l}\text { Overweight } \\
\text { Normal } \\
\text { Underweight }\end{array}$ & $\begin{array}{l}286 \\
411 \\
44\end{array}$ & $\begin{array}{l}38.6 \\
55.5 \\
5.9\end{array}$ \\
\hline Smoking & $\begin{array}{l}\text { Non-smokers } \\
\text { Smokers } \\
\text { Ex-smokers }\end{array}$ & $\begin{array}{l}585 \\
104 \\
52\end{array}$ & $\begin{array}{l}78.9 \\
14 \\
7\end{array}$ \\
\hline Educational level & $\begin{array}{l}\text { Graduate and above } \\
\text { Secondary and higher secondary } \\
\text { Primary and below } \\
\text { Uneducated }\end{array}$ & $\begin{array}{l}122 \\
262 \\
163 \\
194\end{array}$ & $\begin{array}{l}16.5 \\
35.4 \\
22 \\
26.2\end{array}$ \\
\hline Profession & $\begin{array}{l}\text { Officers/higher management } \\
\text { Clerical staff } \\
\text { Workers/labourers } \\
\text { Self-employed } \\
\text { Retired/unemployed } \\
\text { Housewives }\end{array}$ & $\begin{array}{l}78 \\
62 \\
148 \\
15 \\
140 \\
298\end{array}$ & $\begin{array}{l}10.5 \\
8.4 \\
20 \\
2 \\
18.9 \\
40.2\end{array}$ \\
\hline Entitlement status & $\begin{array}{l}\text { Entitled } \\
\text { Non-entitled }\end{array}$ & $\begin{array}{l}303 \\
438\end{array}$ & $\begin{array}{l}40.9 \\
59.1\end{array}$ \\
\hline Treatment duration & $\begin{array}{l}\text { Mean } \pm S D \\
\leq 5 \text { years } \\
6-9 \text { years } \\
\geq 10 \text { years }\end{array}$ & $\begin{array}{l}5.7 \pm 5.9 \\
498 \\
73 \\
170\end{array}$ & $\begin{array}{l}67.2 \\
9.9 \\
22.9\end{array}$ \\
\hline No. of antihypertensive drugs & $\begin{array}{l}\text { Mean } \pm S D \\
1 \\
2 \\
3 \\
4\end{array}$ & $\begin{array}{l}1.7 \pm 0.68 \\
303 \\
340 \\
97 \\
1\end{array}$ & $\begin{array}{l}40.9 \\
45.9 \\
13.1 \\
0.1\end{array}$ \\
\hline Comorbidity & $\begin{array}{l}\text { Yes } \\
\text { No }\end{array}$ & $\begin{array}{l}536 \\
205\end{array}$ & $\begin{array}{l}72.3 \\
27.7\end{array}$ \\
\hline Specific comorbidity & $\begin{array}{l}\text { Patients with DM } \\
\text { Patients with CHD } \\
\text { Patients with CHF } \\
\text { Patients with hyperlipidaemia }\end{array}$ & $\begin{array}{l}125 \\
63 \\
06 \\
169\end{array}$ & $\begin{array}{l}16.9 \\
8.50 \\
0.80 \\
22.8\end{array}$ \\
\hline Blood pressure & $\begin{array}{l}\text { Controlled } \\
\text { Uncontrolled }\end{array}$ & $\begin{array}{l}357 \\
384\end{array}$ & $\begin{array}{l}48.2 \\
51.8\end{array}$ \\
\hline
\end{tabular}

Abbreviations: BMI, body mass index; DM, diabetes mellitus; $\mathrm{CHD}$, coronary heart disease; $\mathrm{CHF}$, congestive heart failure. 
mean systolic blood pressure was $148 \pm 18.6 \mathrm{mmHg}$ and mean diastolic blood pressure was $92 \pm 10.7 \mathrm{mmHg}$. The mean systolic blood pressure was $145 \pm 19 \mathrm{mmHg}$ in patients with good medication adherence, $148 \pm 18 \mathrm{mmHg}$ in patients with moderate adherence and $152 \pm 18 \mathrm{mmHg}$ in patients with low medication adherence. Similarly, the mean diastolic blood pressure was $90 \pm 11 \mathrm{mmHg}$ in patients with good medication adherence, $92 \pm 11 \mathrm{mmHg}$ in patients with moderate adherence and $95 \pm 10 \mathrm{mmHg}$ in patients with low medication adherence (Table 2).

\section{Factors Associated with Medication Adherence}

Patients were categorized into adherent, moderately adherent and non-adherent on the basis of their MMAS-8 score. In terms of level of care, univariate analysis found that patients attending the tertiary care hospital had a higher rate of medication adherence $(169 / 387,43.6 \%)$ compared to secondary care $(79 / 203,38.90 \%)$ and the primary care centre $(36 / 151,23.8 \%)$ (Table 3$)$.

Table 4 shows the results of binary regression analysis for factors predicting medication adherence. Older age (OR 1.783 [95\% CI: 1.172-2.712]), being educated (OR 2.018 [95\% CI: 1.240-3.284]) and longer treatment duration increased the odds of being adherent (OR 2.868 [95\% CI: 1.913-4.299]). Married patients were more likely to be adherent to the antihypertensive therapy than unmarried/ divorced/widowed patients (OR $1.25 \quad[95 \%$ CI:

Table 2 Mean SBP and DBP Stratified by Adherence Level

\begin{tabular}{|l|l|l|}
\hline Adherence Level & $\begin{array}{l}\text { Mean SBP } \\
(\mathbf{m m H g})\end{array}$ & $\begin{array}{l}\text { Mean DBP } \\
(\mathbf{m m H g})\end{array}$ \\
\hline Good adherence & $145 \pm 19$ & $90 \pm 11$ \\
Moderate adherence & $148 \pm 18$ & $92 \pm 11$ \\
Low adherence & $152 \pm 18$ & $95 \pm 10$ \\
\hline
\end{tabular}

Abbreviations: SBP, systolic blood pressure; DBP, diastolic blood pressure.
0.827-1.890]). Patients who were entitled to free medical care were more likely to be adherent to prescribed antihypertensive therapy than patients who were non-entitled (OR 1.369 [95\% CI: 1.009-1.859]). The odds of being adherent increased by 1.973 [95\% CI: 1.560-2.495] times with every unit increase in number of antihypertensive medications. Similarly, patients who had comorbidity were more likely to be adherent to the antihypertensive therapy than the patients who had no comorbidity (OR 2.658 [95\% CI: 1.836-3.848]). Patients with controlled blood pressure were three times more likely to be adherent than those with uncontrolled blood pressure (OR 3.103 [95\% CI: 2.268-4.247]). Male patients were more likely to be adherent than female patients (OR 1.134 [95\% CI: $0.842-1.527])$.

\section{Factors Associated with Optimal Blood Pressure Control}

Univariate analysis revealed that the rate of blood pressure control was $63.7 \%$ among patients who had high medication adherence, compared to $50 \%$ among patients with moderate adherence and $31.2 \%$ among patients who had low adherence to prescribed antihypertensive medication (Table 5). The rate of blood pressure control was $44 \%$ in patients attending the tertiary care hospital, $56 \%$ in patients attending the secondary care hospital and $49 \%$ in patients attending the primary healthcare setting. The overall rate of blood pressure control was $48 \%$ (Table 6).

The results of binary regression analysis showed that age, entitlement status for free medical care, treatment duration, presence of any comorbidity and adherence level had significant associations with controlled blood pressure. The results of binary regression analysis further revealed that the odds of controlled blood pressure were approximately three times greater in patients who were adherent to their prescribed antihypertensive therapy than in patients who were nonadherent (OR 3.103 [95\% CI: 2.268-4.247]). Similarly, the

Table 3 Medication Adherence Level in Different Healthcare Settings

\begin{tabular}{|l|l|l|l|l|}
\hline Healthcare Setting & No. of Patients, N & Adherent, N (\%) & Moderate Adherence, N (\%) & Non-Adherence, N (\%) \\
\hline Tertiary care hospital & 387 & $169(43.66)$ & $90(23.26)$ & $128(33.07)$ \\
Secondary care hospital & 203 & $79(38.90)$ & $47(23.15)$ & $77(37.93)$ \\
Primary care centre & 151 & $36(23.8)$ & $41(27.15)$ & $74(49.0)$ \\
Overall & 741 & $284(38.3)$ & $178(24.0)$ & $279(37.7)$ \\
\hline
\end{tabular}

Notes: The level of adherence was measured through the eight-item Morisky Medication Adherence Scale (MMAS-8). Use of the MMAS is protected by US copyright laws. Permission for use is required. A licensing agreement is available from: Donald E. Morisky, ScD, ScM, MSPH. Use of the @MMAS is protected by US copyright and registered trademark laws. Permission for use is required. A licensing agreement is available from: Donald E. Morisky, 294 Lindura Court, Las Vegas, NV 89I38-4632; dmorisky@gmail. com. The scale's questions are available in the originally published article. ${ }^{23}$ 
Table 4 Binary Logistic Regression Analysis for Factors Predicting Medication Adherence

\begin{tabular}{|c|c|c|c|c|c|}
\hline Parameter & Non-Adherent, N (\%) & Adherent, N (\%) & OR & $\begin{array}{l}\text { 95\% Cl for OR } \\
\text { Lower-Upper }\end{array}$ & $P$-value \\
\hline \multicolumn{6}{|l|}{ Gender } \\
\hline Female & $138(39.2)$ & $214(60.8)$ & I & & \\
\hline Male & $|4|(36.2)$ & $248(63.8)$ & 1.134 & $0.842-1.527$ & 0.407 \\
\hline \multicolumn{6}{|l|}{ Age } \\
\hline $18-40$ years & $68(48.6)$ & $72(5 I .4)$ & 1 & & \\
\hline $4 I-59$ years & $122(35.5)$ & $222(64.5)$ & 1.719 & $1.154-2.559$ & \\
\hline$\geq 60$ years & $89(34.6)$ & $168(65.4)$ & 1.783 & $1.172-2.712$ & $0.013^{*}$ \\
\hline \multicolumn{6}{|l|}{ Marital status } \\
\hline Married & $233(36.9)$ & $399(63.1)$ & 1 & & \\
\hline Unmarried/divorced/widowed & $46(42.2)$ & $63(57.8)$ & 0.800 & $0.529-1.209$ & 0.289 \\
\hline \multicolumn{6}{|l|}{ Educational level } \\
\hline Uneducated & $85(43.8)$ & $109(56.2)$ & 1 & & \\
\hline Primary or below & $65(39.9)$ & $98(60.1)$ & 1.176 & $0.770-1.794$ & \\
\hline Secondary and higher secondary & $95(36.3)$ & $167(63.7)$ & I.37I & $0.938-2.003$ & \\
\hline Graduate and above & $34(27.9)$ & $88(72.1)$ & 2.018 & $1.240-3.284$ & $0.036 *$ \\
\hline \multicolumn{6}{|l|}{ BMI } \\
\hline Obese & $109(38.1)$ & 177 (6I.9) & I & & \\
\hline Normal weight & 157 (38.2) & $254(61.8)$ & 0.996 & $0.730-1.359$ & \\
\hline Underweight & $13(29.5)$ & $31(70.5)$ & 1.468 & $0.736-2.928$ & 0.523 \\
\hline \multicolumn{6}{|l|}{ Profession } \\
\hline Retired/unemployed & $53(37.9)$ & $87(62.1)$ & I & & \\
\hline Housewives & $113(37.9)$ & $185(62.1)$ & 1.003 & $0.663-1.517$ & \\
\hline Officers & $23(29.5)$ & $55(70.5)$ & $\mathrm{I} .46 \mathrm{I}$ & $0.85 I-2.507$ & \\
\hline Clerical staff & $23(37.1)$ & $39(62.9)$ & 1.036 & $0.588-1.824$ & \\
\hline Worker/labourer & $58(39.2)$ & $90(60.8)$ & 0.948 & $0.632-1.420$ & \\
\hline Self-employed & $9(60)$ & $6(40)$ & 0.407 & $0.14 \mid-1.174$ & 0.376 \\
\hline \multicolumn{6}{|l|}{ Entitlement status } \\
\hline Non-entitled & $178(40.6)$ & $260(59.4)$ & I & & \\
\hline Entitled & $101(33.3)$ & $202(66.7)$ & 1.369 & $1.009-1.859$ & $0.044^{*}$ \\
\hline \multicolumn{6}{|l|}{ Smoking status } \\
\hline Non-smoker & $222(37.9)$ & $363(62.1)$ & I & & \\
\hline Smoker & $40(38.5)$ & $64(61.5)$ & 0.979 & $0.637-1.503$ & \\
\hline Ex-smoker & $17(32.7)$ & $35(67.3)$ & 1.259 & $0.689-2.301$ & 0.743 \\
\hline \multicolumn{6}{|l|}{ Treatment duration } \\
\hline$\leq 5$ years & $221(44.4)$ & $277(55.6)$ & I & & \\
\hline $6-9$ years & $21(28.8)$ & $52(7 \mid .2)$ & 1.976 & $1.155-3.379$ & \\
\hline$\geq 10$ years & $37(21.8)$ & $133(78.2)$ & 2.868 & $1.913-4.299$ & $0.000 I^{*}$ \\
\hline \multicolumn{6}{|l|}{ No. of medications } \\
\hline 1 & $15 \mid(49.8)$ & $152(50.2)$ & & & \\
\hline 2 & $107(31.5)$ & $233(68.5)$ & & & \\
\hline 3 & $20(20.6)$ & 77 (79.4) & 1.973 & $1.560-2.495$ & $0.000 I^{*}$ \\
\hline \multicolumn{6}{|l|}{ Comorbidities } \\
\hline No & $233(43.5)$ & $303(56.5)$ & I & & \\
\hline Yes & $46(22.4)$ & $159(77.6)$ & 2.658 & $1.836-3.848$ & $0.000 I^{*}$ \\
\hline
\end{tabular}

(Continued) 
Table 4 (Continued).

\begin{tabular}{|l|l|l|l|l|l|}
\hline Parameter & Non-Adherent, N (\%) & Adherent, N (\%) & OR & $\begin{array}{l}\text { 95\% CI for OR } \\
\text { Lower-Upper }\end{array}$ & \begin{tabular}{l} 
P-value \\
\hline $\begin{array}{l}\text { BP control } \\
\text { Uncontrolled } \\
\text { Controlled }\end{array}$
\end{tabular} \\
\hline
\end{tabular}

Note: $* P<0.05$.

Abbreviations: BMI, body mass index; BP, blood pressure.

Table 5 Adherence Level Among Hypertensive Patients Stratified by Blood Pressure Control

\begin{tabular}{|l|l|l|l|l|}
\hline Adherence Level & Blood Pressure & \multicolumn{3}{|l|}{ Total Study Population } \\
\cline { 3 - 6 } & & $\mathbf{N}$ & $\%$ & Total (\%) \\
\hline High adherence & Controlled & 181 & 63.7 & $284(38.3)$ \\
& Uncontrolled & 103 & 36.3 & $178(24)$ \\
\hline Moderate adherence & Controlled & 89 & 50 & 50 \\
\hline Low adherence & Uncontrolled & 89 & 31.2 & $279(37.7)$ \\
\hline
\end{tabular}

Table 6 Level of Blood Pressure Control in Different Healthcare Settings

\begin{tabular}{|c|c|c|c|c|c|}
\hline Healthcare Setting & Total No. of Patients & BP Controlled BP, N (\%) & $\%$ & Uncontrolled BP, N (\%) & $\%$ \\
\hline Tertiary care hospital & 387 & $169(44)$ & 44 & $218(56)$ & 56 \\
\hline Secondary care hospital & 203 & $113(56)$ & 56 & $90(44)$ & 44 \\
\hline Primary care centre & 151 & $74(49)$ & 49 & $77(5 I)$ & 51 \\
\hline Overall & 741 & $356(48)$ & 48 & $385(52)$ & 52 \\
\hline
\end{tabular}

Abbreviation: BP, blood pressure.

odds of controlled blood pressure were 1.179 times greater in males than in females (OR 1.179 [95\% CI: 0.883-1.574]). Older age ( $\geq 60$ years) (OR 1.998 [95\% CI: 1.313-3.040]), being educated (OR 1.5 [95\% CI: 0.956-2.377]), being an officer (OR 1.5 [95\% CI: 0.923-2.516]) and longer treatment duration (OR 1.4 [95\% CI: 1.010-2.031]) increased the odds of controlled blood pressure. The odds of controlled blood pressure were 1.213 times greater in unmarried/divorced/ widowed patients than in married patients (OR 1.213 [95\% CI: $0.807-1.822]$ ). The odds of controlled blood pressure were 1.5 times greater in patients who had entitlement to free medical care than in who did not have entitlement to free medical care (OR 1.498 [95\% CI: 1.116-2.010]). The number of medications had an inverse relationship with controlled blood pressure with every unit increase in number of medications (OR 0.884 [95\% CI: 0.716-1.091]). The odds of controlled blood pressure were 1.5 times greater in patients who had comorbidity than in patients who had no comorbidity (OR 1.552 [95\% CI: 1.123-2.147]) (Table 7).

\section{Reasons for Non-Adherence/Moderate Adherence}

Multiple reasons for moderate/non-adherence were reported by the patients. Of 457 hypertensive patients, $408(89 \%)$ who had either moderate adherence or nonadherence responded to the question on the reasons for non-adherence. About one-quarter of the patients (24.7\%) responded that they "don't feel a need for regular use" as they take their medicines only when they feel any symptom of hypertension. Carelessness was the second most frequently cited reason for moderate/non-adherence (13.4\%). Adverse effects to antihypertensive medications were also cited by 46 patients (11.2\%) (Table 8 ). 
Table 7 Binary Logistic Regression Analysis for Factors Predicting Controlled Blood Pressure

\begin{tabular}{|c|c|c|c|c|c|}
\hline Parameter & Uncontrolled BP, N (\%) & Controlled BP, N (\%) & OR & 95\% Cl for OR Lower-Upper & $P$-value \\
\hline \multicolumn{6}{|l|}{ Gender } \\
\hline Female & $190(54.0)$ & $162(46.0)$ & 1 & & \\
\hline Male & $194(49.9)$ & $195(50.1)$ & 1.179 & $0.883-1.574$ & 0.264 \\
\hline \multicolumn{6}{|l|}{ Age } \\
\hline $18-40$ years & $86(6 \mid .4)$ & $54(38.6)$ & I & & \\
\hline $4 I-59$ years & $184(53.5)$ & $160(46.6)$ & 1.385 & $0.927-2.068$ & \\
\hline$\geq 60$ years & II 4 (44.4) & $143(55.6)$ & 1.998 & $1.313-3.040$ & $0.004^{*}$ \\
\hline \multicolumn{6}{|l|}{ Marital status } \\
\hline Married & $332(52.5)$ & $300(47.5)$ & I & & \\
\hline Unmarried/divorced/widowed & $52(47.7)$ & $57(52.3)$ & 1.213 & $0.807-1.822$ & 0.352 \\
\hline \multicolumn{6}{|l|}{ Education } \\
\hline Uneducated & $112(57.7)$ & $82(42.3)$ & 1 & & \\
\hline Primary or below & $79(48.5)$ & $84(5 \mid .5)$ & 1.452 & $0.955-2.208$ & \\
\hline $\begin{array}{l}\text { Secondary and higher } \\
\text { secondary }\end{array}$ & $135(5 \mid .5)$ & $127(48.5)$ & 1.285 & $0.884-1.868$ & \\
\hline Graduate and above & $58(47.5)$ & $64(52.5)$ & 1.507 & $0.956-2.377$ & 0.227 \\
\hline \multicolumn{6}{|l|}{ BMI } \\
\hline Obese & $152(53.1)$ & $134(46.9)$ & I & & \\
\hline Normal weight & $216(52.6)$ & $195(47.4)$ & 1.024 & $0.757-1.386$ & \\
\hline Underweight & $16(36.4)$ & $28(63.6)$ & 1.985 & $1.029-3.828$ & 0.113 \\
\hline \multicolumn{6}{|l|}{ Profession } \\
\hline Housewives & $165(55.4)$ & $133(44.6)$ & 1 & & \\
\hline Officers & $35(44.9)$ & $43(55.1)$ & 1.524 & $0.923-2.516$ & \\
\hline Clerical staff & $33(53.2)$ & $29(46.8)$ & 1.090 & $0.630-1.887$ & \\
\hline Worker/labourer & $77(52.0)$ & $71(48.0)$ & I.144 & $0.77 \mid-1.698$ & \\
\hline Retired/unemployed & $65(46.4)$ & $75(53.6)$ & 1.431 & $0.957-2.142$ & \\
\hline Self-employed & $9(60.0)$ & $6(40.0)$ & 0.827 & $0.287-2.382$ & 0.407 \\
\hline \multicolumn{6}{|l|}{ Entitlement status } \\
\hline Non-entitled & $245(55.9)$ & $193(44.1)$ & I & & \\
\hline Entitled & $139(45.9)$ & $164(54.1)$ & 1.498 & $1.116-2.010$ & $0.007 *$ \\
\hline \multicolumn{6}{|l|}{ Smoking status } \\
\hline Non-smoker & $301(5 I .5)$ & $284(48.5)$ & I & & \\
\hline Smoker & $58(55.8)$ & $46(44.2)$ & $0.84 I$ & $0.553-1.279$ & \\
\hline Ex-smoker & $25(48.1)$ & $27(51.9)$ & 1.145 & $0.649-2.019$ & 0.615 \\
\hline \multicolumn{6}{|l|}{ Treatment duration } \\
\hline$\leq 5$ years & $276(55.4)$ & $222(44.6)$ & I & & \\
\hline 6-9 years & $29(39.7)$ & $44(60.3)$ & 1.886 & $1.143-3.113$ & \\
\hline$\geq 10$ years & $79(46.5)$ & 91 (53.5) & 1.432 & $1.010-2.031$ & $0.013^{*}$ \\
\hline \multicolumn{6}{|l|}{ No. of medications } \\
\hline I & $156(5 \mid .5)$ & $147(48.5)$ & & & \\
\hline 2 & $168(49.4)$ & $172(50.6)$ & & & \\
\hline 3 & $59(60.8)$ & $38(39.2)$ & 0.884 & $0.716-1.091$ & 0.250 \\
\hline \multicolumn{6}{|l|}{ Comorbidities } \\
\hline No & $294(54.9)$ & $242(45.1)$ & I & & \\
\hline Yes & $90(43.9)$ & II $5(56.1)$ & 1.552 & $1.123-2.147$ & $0.008^{*}$ \\
\hline
\end{tabular}

(Continued) 
Table 7 (Continued).

\begin{tabular}{|l|l|l|l|l|l|}
\hline Parameter & Uncontrolled BP, N (\%) & Controlled BP, N (\%) & OR & 95\% CI for OR Lower-Upper & P-value \\
\hline $\begin{array}{l}\text { Medication adherence level } \\
\text { Non-adherent }\end{array}$ & & & & & \\
Adherent & $192(68.8)$ & $87(31.2)$ & 1 & & \\
\end{tabular}

Note: $* P<0.05$.

Abbreviations: BMI, body mass index; BP, blood pressure.

Table 8 Reasons “In Patient's View” for Either Non-Adherence or Moderate Adherence to Prescribed Antihypertensive Therapy

\begin{tabular}{|l|l|l|}
\hline \multirow{2}{*}{ Sr. } & Reason & \multirow{2}{*}{ n (\%) } \\
\cline { 2 - 2 } & No. of Respondents (n=408) & \\
\hline 1 & Don't feel need for regular use & $101(24.7)$ \\
2 & Carelessness & $55(13.4)$ \\
3 & Adverse effect & $46(11.2)$ \\
4 & Forgetfulness & $40(9.8)$ \\
5 & Lack of education & $40(9.8)$ \\
6 & Travelling & $35(8.6)$ \\
7 & Tired of taking medication & $31(7.6)$ \\
8 & Fear of addiction & $24(5.9)$ \\
9 & Unaffordable & $19(4.7)$ \\
10 & Unavailability & $16(3.9)$ \\
\hline
\end{tabular}

\section{Discussion}

Medication adherence, predictors of medication adherence and its association with controlled blood pressure, as well as the reasons for medication non-adherence, were investigated in this study. Patient groups with poor blood pressure control were identified. This study could potentially help medical practitioners to design patient-specific interventions targeting these patients, including patient counselling, providing entitlement to free medical care and helping policy makers develop policies to ensure better therapeutic outcomes. Overall, 37.7\% of hypertensive patients attending primary, secondary and tertiary healthcare settings in Islamabad were non-adherent. There was a significant association between medication adherence and blood pressure control. "Don't feel need for regular use", "Carelessness" and "adverse effects" were the main reasons for non-adherence among hypertensive patients.

Previous studies conducted in Pakistan have reported higher rates of non-adherence to antihypertensive therapy, ranging between $51 \%$ and $82 \%{ }^{18,19}$, compared to our study. This difference may be due to the use of unvalidated questionnaires to assess medication adherence and very small sample sizes ( 89 and 32). The use of unvalidated questionnaires may have resulted in overestimation of nonadherence. However, one study, conducted in the metropolitan city of Karachi, Pakistan, ${ }^{17}$ was the only research study that used a validated questionnaire, ie the Morisky, Green, Levine Medication Adherence Questionnaire. Our study reported less medication adherence than was reported in the previous study conducted in Karachi, ie $77 \%$. This difference may be due to the different methods used to estimate adherence, as it has been reported in a meta-analysis that MMAS- 8 reports less adherence compared to other questionnaires used for the estimation of medication adherence. This is because MMAS- 8 makes it possible to obtain a more in-depth picture of adherence, where the participants do not feel forced to claim to be adherent. ${ }^{28}$ Various studies conducted in different Asian countries, ie China, Hong Kong, Saudi Arabia, UAE and Iran, using MMAS-8 have also reported similar poor adherence to prescribed antihypertensive therapy, supporting our results. $^{15,29-35}$

The WHO estimates the prevalence of non-adherence among patients with chronic diseases to be between $52 \%$ and $74 \%$, defined by $\geq 80 \%$ cut-off. ${ }^{36}$

In this study, we found no significant correlation between gender and medication adherence. This finding is consistent with previously reported studies, including a study in Pakistan. ${ }^{17,30,31,37,38}$ In contrast, there are studies that have found a significant correlation between gender and medication adherence. ${ }^{29,32}$ Age was found to be significantly associated with medication adherence in our study, with better adherence observed in older patients. This finding is consistent with another study from Pakistan, ${ }^{17}$ as well as with a number of international studies. ${ }^{29,39-42}$ However, other studies have shown either no correlation with age ${ }^{43,44}$ or an inverse correlation with increasing age when it comes to the use of antihypertensives. ${ }^{45}$

In our opinion, this negative correlation may be due to loss of memory, non-availability of any caregiver or deteriorated health of the patients. On the other hand, the increased medication adherence in older patients may be due to either increased severity of the condition, which forced the patient to be adherent, or strong family support 
for older people in Pakistani society. Studies have reported a positive relationship between family support and medication adherence. ${ }^{46,47}$

The level of education of the participants was also found to be significantly associated with medication adherence in our study. There was a positive correlation between medication adherence and the level of education. This is in contrast to what has been reported previously in studies conducted in different Asian countries, as most of the previous studies using MMAS- 8 have found no significant correlation between level of education and medication adherence. $^{17,29-31,37}$ Increased medication adherence in educated patients may be due to increased awareness about the disease. This is in line with previous research which showed the significant relationship between formal education and medication adherence. ${ }^{48,49}$

The number of medications had a significant correlation with medication adherence. Medication adherence increases when the patient is using more than one medication to control blood pressure. This finding is in contrast to previously reported studies, which found no significant correlation between number of medications and medicationadherence. $^{17,29-31,37,38}$ In our opinion, the increased medication adherence in patients who use more than one medication to control their blood pressure may be due to an increased severity of symptoms in these patients, which compels these patients to remain adherent with their prescribed medications.

Similarly, presence of comorbidity also had strong a correlation with medication adherence. Mixed findings have been reported in the literature regarding the association between presence of comorbidity and medication adherence. Some studies support our finding, ${ }^{29,38}$ whereas other studies found no significant correlation between presence of comorbidity and medication adherence. ${ }^{17,31,37}$ No significant correlation was found between gender, marital status, BMI, smoking or employment status and medication adherence.

For blood pressure control, the age of the patient was found to have a significant correlation with controlled blood pressure. We found that patients aged $\geq 60$ years had better blood pressure control than patients who were under 60 years of age. This finding is in contrast to previously reported studies, which found the opposite result. $^{29,50}$ This better control of blood pressure could be due to better medication adherence in elderly patients and increased self-care in old age.
This study also highlighted various self-reported reasons for medication non-adherence/moderate adherence among Pakistani patients. These reasons included "no need for regular use", carelessness, adverse effects of medication, forgetfulness, lack of education, travelling, tired of taking medicines, fear of addiction, unaffordability and unavailability. A previous study, also conducted in Pakistan, reported misperception, no need for medications, ineffectiveness, forgetfulness, unavailability and adverse effects of medications as the main reasons for nonadherence in hypertensive patients. ${ }^{19}$ A study conducted in India revealed that forgetfulness, blood pressure assumed to be normal and non-availability were the main reasons for non-adherence. ${ }^{51}$ Similarly, a study conducted in Bangladesh reported that forgetfulness, feeling well/no need for medication use and busy schedule were the three main reasons for non-adherence. ${ }^{52}$ In another study conducted in Bangladesh, forgetfulness, busy schedule and boredom were reported as the three main factors for medication non-adherence in hypertensive patients. ${ }^{53}$ All of these South Asian studies showed that forgetfulness, feeling well/no need for medicines were the major reasons for medication non-adherence in hypertensive patients.

\section{Implications for Clinical Practice}

The findings of this study have critical implications for healthcare practitioners and policy makers. There is a clear need to design and implement different interventions with the aim of improving medication adherence among patients. In this study, we have identified different predictors for medication adherence, such as age, level of education, entitlement/insurance status, treatment duration, number of antihypertensive medications and presence of comorbidities. Hence, any intervention targeting any of these predictors could be helpful in improving medication adherence. Effective strategies to improve medication adherence can be achieved through better communications between the physician and patient and can help to improve medication adherence. Interventions have to be patient specific in order to be successful. Patient-related factors such as gender, culture, health beliefs and knowledge about disease should be kept in mind when designing any intervention for improving medication adherence. Limited local evidence is available on the effectiveness of interventions to improve medication adherence among hypertensive patients in Pakistan. However, internationally, effective interventions to improve medication adherence include educational programmes involving both 
patients and family members, in the form of individual instructions or group classes; regular monitoring of blood pressure at home; providing entitlement to free medical care; simplifying the dosing regimen; and behavioural interventions, such as memory aids and synchronizing therapeutic activities with the daily routine, eg taking medications before a shower or after prayer. ${ }^{54,55}$ The existing literature has demonstrated that interventions aimed at improving health literacy, using communication technologies such as mobile applications and sending SMS reminders, electronic medication reconciliation and motivational interviewing can improve medication adherence and may have positive effects on treatment outcomes. ${ }^{56-63}$

\section{Study Limitations}

There are a few limitations to our study findings. This study was conducted in only one city in Pakistan; therefore, the findings may not be generalizable to other cities, towns and villages. Self-administered questionnaires were used in this research to assess medication adherence, which may lead to under- or overestimation of results. This questionnaire required recall of previous events, which may lead to recall bias, especially in older patients or those who had been on antihypertensive treatment for many years. However, MMAS-8 has very well-documented reliability and validity in assessing medication adherence. In this study, comorbidity was recorded as a dichotomous outcome (present/not present); however, the number and nature of comorbidities, which could potentially affect medication adherence and treatment outcome, were not recorded. Future studies should collect data focusing on the number and nature of comorbidities, and their impact on medication adherence.

\section{Conclusion}

Adherence to prescribed antihypertensive medications was suboptimal in hypertensive patients attending different healthcare settings in Islamabad, Pakistan. This suboptimal medication adherence was associated with poor blood pressure control. Keeping in view the predictors of good blood pressure control identified in this study, targeted interventions should be introduced and implemented for better treatment outcomes.

\section{Acknowledgments}

The authors thank Professor Donald E. Morisky, Department of Community Health Sciences, UCLA Fielding School of Public Health, Los Angeles, United States, for granting us permission to use the copyrighted
MMAS-8. Use of the CMMAS is protected by US copyright and registered trademark laws. Permission for use is required. A licensing agreement is available from: Donald E. Morisky, 294 Lindura Court, Las Vegas, NV 891384632; dmorisky@gmail.com.

\section{Disclosure}

The authors declare that they have no conflicts of interest.

\section{References}

1. Kokubo Y, Iwashima Y. Higher blood pressure as a risk factor for diseases other than stroke and ischemic heart disease. Hypertension. 2015;66(2):254-259. doi:10.1161/HYPERTENSIONAHA.115.03480

2. Rapsomaniki E, Timmis A, George J, et al. Blood pressure and incidence of twelve cardiovascular diseases: lifetime risks, healthy life-years lost, and age-specific associations in 1. 25 million people. Lancet. 2014;383(9932):1899-1911. doi:10.1016/S0140-6736(14) 60685-1

3. Mills KT, Bundy JD, Kelly TN, et al. Global burden of hypertension: analysis of population-based studies from 89 countries. J Hypertens. 2015;33:e2. doi:10.1097/01.hjh.0000469726.59998.cc

4. W.H.O report on global prevalence of hypertension published on World Health Day; 2013. Available from: https://apps.who.int/iris/ bitstream/handle/10665/79059/WHO_DCO_WHD_2013.2_eng.pdf; jsessionid=F638668E0D914847E4B72CCAFB90BC63? sequence $=1$. Accessed July 27, 2019.

5. Mohan S, Campbell NR. Hypertension management. Hypertension. 2009;53(3):450-451. doi:10.1161/HYPERTENSIONAHA.108.127076

6. Prenissl J, Manne-Goehler J, Jaacks LM, et al. Hypertension screening, awareness, treatment, and control in India: a nationally representative cross-sectional study among individuals aged 15 to 49 years. PLoS Med. 2019;16(5):e1002801. doi:10.1371/journal.pmed.1002801

7. Lu J, Lu Y, Wang X, et al. Prevalence, awareness, treatment, and control of hypertension in China: data from 1. 7 million adults in a population-based screening study (China PEACE million persons project). Lancet. 2017;390(10112):2549-2558. doi:10.1016/S01406736(17)32478-9

8. Shah N, Shah Q, Shah AJ. The burden and high prevalence of hypertension in Pakistani adolescents: a meta-analysis of the published studies. Arch Public Health. 2018;76(1):20. doi:10.1186/ s13690-018-0265-5

9. Khatib R, Schwalm J-D, Yusuf S, et al. Patient and healthcare provider barriers to hypertension awareness, treatment and follow up: a systematic review and meta-analysis of qualitative and quantitative studies. PLoS One. 2014;9(1):e84238. doi:10.1371/journal. pone. 0084238

10. Jaam M, Hadi MA, Kheir N, et al. A qualitative exploration of barriers to medication adherence among patients with uncontrolled diabetes in Qatar: integrating perspectives of patients and health care providers. Patient Prefer Adherence. 2018;12:2205. doi:10.2147/ PPA.S174652

11. Jaam M, Ibrahim MIM, Kheir N, Hadi MA, Diab MI, Awaisu A. Assessing prevalence of and barriers to medication adherence in patients with uncontrolled diabetes attending primary healthcare clinics in Qatar. Prim Care Diabetes. 2018;12(2):116-125. doi:10.1016/j.pcd.2017.11.001

12. Cramer J, Benedict A, Muszbek N, Keskinaslan A, Khan Z. The significance of compliance and persistence in the treatment of diabetes, hypertension and dyslipidaemia: a review. Int $J$ Clin Pract. 2008;62(1):76-87. doi:10.1111/j.1742-1241.2007.01630.x 
13. Levy G, Zamacona MK, Jusko WJ. Developing compliance instructions for drug labeling. Clin Pharmacol Ther. 2000;68(6):586-591. doi: $10.1067 / \mathrm{mcp} .2000 .110976$

14. Gibaldi M. Failure to comply: a therapeutic dilemma and the bane of clinical trials. J Clin Pharmacol. 1996;36(8):674-682. doi:10.1002/ jcph.1996.36.issue-8

15. Li YT, Wang HH, Liu KQ, et al. Medication adherence and blood pressure control among hypertensive patients with coexisting long-term conditions in primary care settings: a cross-sectional analysis. Medicine. 2016;95 (20). doi:10.1097/MD.0000000000004864.

16. Jalal Z, Antoniou S, Taylor D, Paudyal V, Finlay K, Smith F. South Asians living in the UK and adherence to coronary heart disease medication: a mixed-method study. Int J Clin Pharm. 2019;41 (1):122-130. doi:10.1007/s11096-018-0760-3

17. Hashmi SK, Afridi MB, Abbas K, et al. Factors associated with adherence to anti-hypertensive treatment in Pakistan. PLoS One. 2007;2(3):e280. doi:10.1371/journal.pone.0000280

18. Ahmed N, Abdul Khaliq M, Shah SH, Anwar W. Compliance to antihypertensive drugs, salt restriction, exercise and control of systemic hypertension in hypertensive patients at Abbottabad. J Ayub Med Coll Abbottabad. 2008;20(2):66-69.

19. Ali M, Hameed J, Zia MH, Masood RA, Shahzad A. Patient compliance in systemic hypertension and to identify causes of non-compliance. J Islam Int Med Coll. 2013:48.

20. Morisky DE, Green LW, Levine DM. Concurrent and predictive validity of a self-reported measure of medication adherence. Med Care. 1986;24:67-74. doi:10.1097/00005650-198601000-00007

21. Meghani ST, Sehar S, Punjani NS. Comparison and analysis of health care delivery system: pakistan versus China. Int J Endorsing Health Sci Res. 2014;2:46-50.

22. Islam A. Health sector reform in Pakistan: future directions. J Pak Med Assoc. 2002;52(4):174-182.

23. Morisky DE, Ang A, Krousel-Wood M, Ward HJ. Predictive validity of a medication adherence measure in an outpatient setting. $J$ Clin Hypertens. 2008;10(5):348-354. doi:10.1111/j.1751-7176.2008.075 72.x

24. Berlowitz DR, Foy CG, Kazis LE, et al. Effect of intensive blood-pressure treatment on patient-reported outcomes. $N \mathrm{Engl}$ $J$ Med. 2017;377(8):733-744. doi:10.1056/NEJMoa1611179

25. Morisky DE, DiMatteo MR. Improving the measurement of self-reported medication nonadherence: response to authors. J Clin Epidemiol. 2011;64(3):255-257. doi:10.1016/j.jclinepi.2010. 09.002

26. Saleem F, Hassali MA, Akmal S, et al. Translation and validation study of Morisky Medication Adherence Scale (MMAS): the Urdu version for facilitating person-centered healthcare in Pakistan. Int J Pers Cent Med. 2012;2(3):384-390.

27. National Institute for Health and Care Excellence (NICE) [Internet]. Manchester, UK: Hypertension in adults: diagnosis and management NICE guidelines [CG127]; August 2011 [reviewed October 2013]. Available from: https://www.nice.org.uk/guidance/cg127. Accessed December 31, 2019.

28. Nielsen JØ, Shrestha AD, Neupane D, Kallestrup P. Non-adherence to anti-hypertensive medication in low-and middle-income countries: a systematic review and meta-analysis of 92443 subjects. J Hum Hypertens. 2017;31(1):14. doi:10.1038/jhh.2016.31

29. Khayyat SM, Khayyat SMS, Alhazmi RSH, Mohamed MM, Hadi MA. Predictors of medication adherence and blood pressure control among Saudi hypertensive patients attending primary care clinics: a cross-sectional study. PLoS One. 2017;12(1):e171255. doi:10.1371/journal.pone.0171255

30. Lee GK, Wang HH, Liu KQ, Cheung Y, Morisky DE, Wong MC. Determinants of medication adherence to antihypertensive medications among a Chinese population using Morisky medication adherence scale. PLoS One. 2013;8(4):e62775. doi:10.1371/journal. pone. 0062775
31. Kang CD, Tsang PP, Li WT, et al. Determinants of medication adherence and blood pressure control among hypertensive patients in Hong Kong: a cross-sectional study. Int $J$ Cardiol. 2015;182:250-257. doi:10.1016/j.ijcard.2014.12.064

32. Han WP, Hong SA, Tiraphat S. Factors related to medication adherence among essential hypertensive patients in tertiary hospitals in Yangon, Myanmar. J Public Health Dev. 2015;13(2):43-56.

33. Shaik SA, Alsuwailem A, Alhargan A, et al. Medications adherence level and its associated factors among hypertensive patients at a major referral hospital, in Riyadh, KSA. Asian J Med Sci. 2016;7 (4):24-30. doi:10.3126/ajms.v7i4.14085

34. Fahey M, Abdulmajeed A, Sabra K. Measurement of adherence to anti-hypertensive medication as perceived by doctors and patients. Qatar Med J. 2006;2006(1):16. doi:10.5339/qmj.2006.1.16

35. Saadat Z, Nikdoust F, Aerab-Sheibani H, et al. Adherence to antihypertensives in patients with comorbid condition. Nephrourol Mon. 2015;7(4). doi:10.5812/numonthly.

36. Organization WH. Adherence to long-term therapies: evidence for action. 2003. https://apps.who.int/iris/bitstream/handle/10665/42682/ 9241545992.pdf. Accessed July 27, 2019.

37. Yue Z, Li C, Weilin Q, Bin W. Application of the health belief model to improve the understanding of antihypertensive medication adherence among Chinese patients. Patient Educ Couns. 2015;98 (5):669-673. doi:10.1016/j.pec.2015.02.007

38. Al-Ramahi R. Adherence to medications and associated factors: a cross-sectional study among Palestinian hypertensive patients. $J$ Epidemiol Glob Health. 2015;5(2):125-132. doi:10.1016/j. jegh.2014.05.005

39. Hassan N, Hasanah C, Foong K, et al. Identification of psychosocial factors of noncompliance in hypertensive patients. J Hum Hypertens. 2006;20(1):23. doi:10.1038/sj.jhh.1001930

40. Caro JJ, Salas M, Speckman JL, Raggio G, Jackson JD. Persistence with treatment for hypertension in actual practice. CMAJ. 1999;160(1):31-37.

41. Monane M, Bohn RL, Gurwitz JH, Glynn RJ, Levin R, Avorn J. Compliance with antihypertensive therapy among elderly Medicaid enrollees: the roles of age, gender, and race. Am J Public Health. 1996;86(12):1805-1808. doi:10.2105/AJPH.86.12.1805

42. Krousel-Wood M, Thomas S, Muntner P, Morisky D. Medication adherence: a key factor in achieving blood pressure control and good clinical outcomes in hypertensive patients. Curr Opin Cardiol. 2004;19(4):357-362. doi:10.1097/01.hco.0000126978.03828.9e

43. Inkster ME, Donnan P, MacDonald T, Sullivan F, Fahey $T$. Adherence to antihypertensive medication and association with patient and practice factors. J Hum Hypertens. 2006;20(4):295. doi:10.1038/sj.jhh.1001981

44. Youssef R, Moubarak I. Patterns and determinants of treatment compliance among hypertensive patients. 2002.

45. Gryglewska B. How can we improve the effectiveness of treatment in elderly hypertensives? Blood Press. 2005;14(sup2):46-49. doi:10.1080/08038020500428740

46. Olowookere AJ, Olowookere SA, Talabi AO, Etonyeaku AC, Adeleke OE, Akinboboye OO. Perceived family support and factors influencing medication adherence among hypertensive patients attending a Nigerian tertiary hospital. Ann Trop Med Public Health. 2015;8(6):241. doi:10.4103/1755-6783.162668

47. Marín-Reyes F, Rodríguez-Morán M. Family support of treatment compliance in essential arterial hypertension. Salud Publica Mex. 2001;43(4):336-339. doi:10.1590/S0036-36342001000400010

48. Setiati S, Sutrisna B. Prevalence of hypertension without anti-hypertensive medications and its association with social demographic characteristics among 40 years and above adult population in Indonesia. Acta Med Indones. 2005;37(1):20-25.

49. Taibanguay N, Chaiamnuay S, Asavatanabodee P, Narongroeknawin P. Effect of patient education on medication adherence of patients with rheumatoid arthritis: a randomized controlled trial. Patient Prefer Adherence. 2019;13:119. doi:10.2147/PPA.S192008 
50. Jahangiry L, Ghanbari J, Farhangi MA, Sarbakhsh P, Ponnet K. Predictors of poor blood pressure control among Iranian hypertensive patients. BMC Res Notes. 2017;10(1):668. doi:10.1186/s13104-017-2971-4

51. Mallya SD, Kumar A, Kamath A, Shetty A, Mishra S. Assessment of treatment adherence among hypertensive patients in a coastal area of Karnataka, India. Int J Community Med Public Health. 2017;3 (8):1998-2003.

52. Hussanin S, Boonshuyar C, Ekram A. Non-adherence to antihypertensive treatment in essential hypertensive patients in Rajshahi, Bangladesh. Anwer Khan Mod Med Coll J. 2011;2(1):9-14. doi:10.3329/akmmcj.v2i1.7465

53. Ekram AS, Hussain SM, Boonshuyar C. Preliminary report on non-adherence to antihypertensive treatment in essential hypertensive patients: a community based survey. J Teachers Assoc. 2008;21 (2):112-117.

54. Fenerty SD, West C, Davis SA, Kaplan SG, Feldman SR. The effect of reminder systems on patients' adherence to treatment. Patient Prefer Adherence. 2012;6:127.

55. Jin J, Sklar GE, Oh VMS, Li SC. Factors affecting therapeutic compliance: a review from the patient's perspective. Ther Clin Risk Manag. 2008;4(1):269. doi:10.2147/TCRM.S1458

56. Gwadry-Sridhar FH, Manias E, Lal L, et al. Impact of interventions on medication adherence and blood pressure control in patients with essential hypertension: a systematic review by the ISPOR medication adherence and persistence special interest group. Value Health. 2013;16(5):863-871. doi:10.1016/j.jval.2013.03.1631

57. Conn VS, Ruppar TM, Chase J-AD, Enriquez M, Cooper PS. Interventions to improve medication adherence in hypertensive patients: systematic review and meta-analysis. Curr Hypertens Rep. 2015;17(12):94. doi:10.1007/s11906-015-0606-5
58. Palacio AM, Uribe C, Hazel-Fernandez L, et al. Can phone-based motivational interviewing improve medication adherence to antiplatelet medications after a coronary stent among racial minorities? A randomized trial. J Gen Intern Med. 2015;30(4):469-475. doi:10.1007/s11606-0143139-8

59. Kamal AK, Muqeet A, Farhat K, et al. Using a tailored health information technology-driven intervention to improve health literacy and medication adherence in a Pakistani population with vascular disease (Talking $\mathrm{Rx}$ )-study protocol for a randomized controlled trial. Trials. 2016;17(1):121. doi:10.1186/s13063-0161244-1

60. Zomahoun HTV, Guenette L, Gregoire J-P, et al. Effectiveness of motivational interviewing interventions on medication adherence in adults with chronic diseases: a systematic review and meta-analysis. Int $J$ Epidemiol. 2016;46(2):589-602.

61. Vollmer WM, Feldstein A, Smith D, et al. Use of health information technology to improve medication adherence. Am J Manag Care. 2011;17(12):SP79.

62. Jalal ZS, Smith F, Taylor D, Patel H, Finlay K, Antoniou S. Pharmacy care and adherence to primary and secondary prevention cardiovascular medication: a systematic review of studies. Eur J Hosp Pharm. 2014;21(4):238-244. doi:10.1136/ejhpharm-2014000455

63. Jalal ZS, Smith F, Taylor D, Finlay K, Patel H, Antoniou S. Impact of pharmacy care upon adherence to cardiovascular medicines: a feasibility pilot controlled trial. Eur J Hosp Pharm. 2016;23 (5):250-256. doi:10.1136/ejhpharm-2015-000790
Patient Preference and Adherence

\section{Publish your work in this journal}

Patient Preference and Adherence is an international, peer-reviewed, open access journal that focusing on the growing importance of patient preference and adherence throughout the therapeutic continuum. Patient satisfaction, acceptability, quality of life, compliance, persistence and their role in developing new therapeutic modalities and compounds to optimize clinical outcomes for existing disease states are major areas of interest for the journal. This journal has been accepted for indexing on PubMed Central. The manuscript management system is completely online and includes a very quick and fair peer-review system, which is all easy to use. Visit http:// www.dovepress.com/testimonials.php to read real quotes from published authors. 